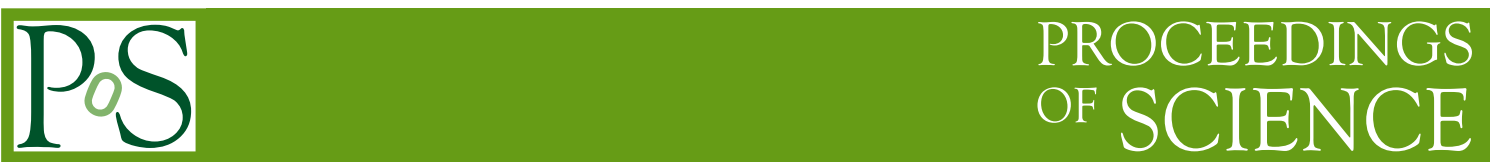

\title{
VHE gamma-ray emission from radio galaxies
}

\section{A. Prokhorov (for the H.E.S.S collaboration)*}

School of Physics, University of the Witwatersrand, Johannesburg, Wits 2050, South Africa

E-mail: dmitry.prokhorov@wits.ac.za

Centaurus A (Cen A), the nearest radio galaxy, was detected as a faint emitter of very high energy (VHE) gamma rays by the H.E.S.S. telescopes in Namibia. The flux derived from the H.E.S.S. data is notably higher than that expected from a single zone synchrotron self-Compton model, which adequately describes the emission from Cen A at lower energies. New observations with H.E.S.S. were performed to clarify the spectral characteristics of the VHE emission from Cen A. I will report on the results of the analysis of the complete H.E.S.S. data set with twice the exposure time of the previously published data set and also the update of the Cen A spectrum obtained with Fermi-LAT at GeV energies. To conclude I will discuss other recent results of, and prospects for, H.E.S.S. observations of the radio galaxies, PKS 0625-35 and Fornax A, respectively.

High Energy Astrophysics in Southern Africa (HEASA 2018)

1-3 August, 2018

Parys, Free State, South Africa

${ }^{*}$ Speaker. 


\section{Introduction}

Very high energy (VHE; $>100 \mathrm{GeV}$ ) observations of active galactic nuclei (AGN) are an important tool to probe the physics of extreme environments. Radio-loud AGN characterised by prominent radio jet and/or lobe emission include both radio galaxies and blazars. Jet formation is likely to be closely connected with the central supermassive black hole in AGN, while the lobes are believed to be inflated by the jets. At present, radio-loud AGN make up $40 \%$ of the VHE sources detected with ground-based imaging atmospheric Cherenkov telescopes [1]. Most of these VHE detected AGN are of the blazar type, i.e. objects where the jet is thought to be inclined at very small viewing angles to the line of sight to Earth. Thus, blazars are a sub-class of a larger parent population of radio galaxies, distinguished by a close alignment of their jets with the line of sight. This alignment results in substantial Doppler-boosting of their intrinsic jet emission privileging the detection of blazars. Nevertheless, non-blazar AGN such as radio galaxies, while less luminous, have emerged as a new gamma-ray emitting source class. With their jets misaligned and associated Doppler boosting effects modest, radio galaxies provide us with unique insights into regions hidden in the case of blazars by emission from the jet and into physical processes occurring in these regions (for a review, see [2]).

Six radio galaxies are established as VHE emitters, see references in [2]. Among these six radio galaxies, Centaurus $\mathrm{A}$ (Cen $\mathrm{A}$; at the distance of $3.8 \mathrm{Mpc}$, the nearest radio galaxy), M 87 (at the distance of $16 \mathrm{Mpc}$, the second nearest radio galaxy), and PKS 0625-35 (at the distance of $220 \mathrm{Mpc}$, the furthest known VHE-emitting radio galaxy) have been discovered at VHE energies with the High Energy Stereoscopic System (H.E.S.S.) located in Namibia. The other three radio galaxies detected at VHE, NGC 1275, IC 310, and 3C 264, are observing targets suited for northern ground-based Cherenkov telescopes. These six radio galaxies are amongst two dozen radio galaxies detected in the high-energy (HE; $100 \mathrm{MeV}-100 \mathrm{GeV}$ ) gamma-ray band with the Fermi Large Area Telescope (LAT). Another nearby radio galaxy detected in the HE gamma-ray band with LAT, Fornax A, located at a distance of $19 \mathrm{Mpc}$ is one of the most promising radio galaxies with respect to detectability with H.E.S.S.. In this invited contribution, I will review recent results of the H.E.S.S. observations of Centaurus A and PKS 0625-35, and outline the potential of H.E.S.S. observations of Fornax A. Given the locations of these three radio galaxies in the southern hemisphere sky, the H.E.S.S. telescope is the only one of the modern ground-based Cherenkov telescopes allowing these VHE studies. In 2018, new papers by the H.E.S.S. collaboration were published regarding Cen A [3] and PKS 0625-35 [4]. The former paper confirmed the previous detection of the Cen A gamma-ray core with H.E.S.S. [5] at a unprecedented level and provided evidence for a new gamma-ray emitting component connecting the high-energy emission above the break energy at a few $\mathrm{GeV}$ to the one observed at VHE energies. Given the angular resolution of H.E.S.S. and FermiLAT, the large-scale jet and inner lobes of Cen A could in principle also contribute to the observed gamma-ray signal from the gamma-ray core. The latter paper presented the VHE discovery of PKS 0625-35 and the possible scenarios responsible for the observed emission. With the aim of studying the Fornax A lobes at VHE, the observing proposal has been submitted by researchers from University of the Witwatersrand (myself and Prof. Sergio Colafrancesco ${ }^{1}$ ) to an observing committee in order to accumulate H.E.S.S. data towards Fornax A.

\footnotetext{
${ }^{1}$ Dr. Dmitry A. Prokhorov honors the memory of Prof. Sergio Colafrancesco, who was a co-author of the proposal.
} 
Based on their radio properties, the radio galaxies detected at VHE have been classified as radio galaxies of Fanaroff-Riley type I. According to AGN unification schemes, radio galaxies of this type are thought to correspond to BL Lacertae (BL Lac) objects viewed from the side, the latter correspond to a subclass of blazars [6]. BL Lac objects are the most abundant class of known extragalactic VHE AGN and exhibit double-peaked spectral energy distributions (SEDs). Their low-frequency emission in the radio to ultraviolet (and X-ray, for high-peaked BL Lacs) band is likely owing to synchrotron emission from relativistic electrons within a blob (zone) moving at relativistic speeds in the jet. The high-energy emission (hard X-ray to VHE gamma-ray) from high-peaked BL Lac type objects has been satisfactorily modelled as synchrotron self-Compton (SSC) radiation resulting from the inverse Compton upscattering of synchrotron photons by the same relativistic electron population that produced the synchrotron radiation, although other more complex models (involving external inverse Compton emission, hadronic interactions, or multiple zones) are possible [7].

In the first part of this paper I will present the results of the H.E.S.S. observations of the Cen A gamma-ray core. To obtain these results, we included more than hundred hours of new H.E.S.S. observations of this source and applied advanced analysis methods. On the basis of 213 hours of the H.E.S.S. observations and 8 years of Fermi-LAT observations, and in collaboration with the Fermi-LAT team we found that the spectrum of the Cen A gamma-ray core hardens at $\mathrm{GeV}$ energies and extends into the VHE regime [3] supporting the result by Sahakyan et al. [8] based on four years of Fermi-LAT data. These spectral properties are suggestive of a new gamma-ray component in the emission from the unresolved Cen A gamma-ray core. A variety of scenarios for its origin are conceivable, but the absence of significant variability at both $\mathrm{GeV}$ and $\mathrm{TeV}$ energies has not allowed yet to disentangle the physical nature of this component. However, a single-zone SSC interpretation of the SED of the Cen A gamma-ray core is disfavoured by these observations.

In the second part I will focus on PKS 0625-35 and Fornax A. I will give a summary of the VHE results on the radio galaxy PKS 0625-35 and discuss the possible origin of its VHE emission. This radio galaxy is located at the centre of the galaxy cluster Abell 3392 and is one of the gammaray-emitting radio galaxies at the centres of galaxy clusters along with M 87 (in the Virgo cluster) and NGC 1275 (in the Perseus cluster). However, PKS 0625-35 is three times more distant than NGC 1275 and, if it is un-beamed, would require a considerably higher luminosity than that of the other radio galaxies detected at VHE. I will then briefly discuss the potential for VHE studies of Fornax A with H.E.S.S. and to study giant lobes of radio galaxies in the VHE band.

\section{Centaurus A gamma-ray core}

\subsection{H.E.S.S. observations and results}

The observations of Cen A with H.E.S.S. reported in the paper [3] were performed in wobble mode, that is with the target typically offset by about $0.5^{\circ}$ or $0.7^{\circ}$ from the pointing direction, allowing simultaneous background estimation in the same field of view. The data were recorded in 28-minute exposures, called runs, which are chosen to minimise systematic changes in instrumental response. The observations of Cen A were carried out during the January-July visibility window. At the time of the observations between 2004 and 2010, the H.E.S.S. array consisted of four 12metre telescopes. 
The first data set A (set A) was taken between April 2004 and July 2008, and $111 \mathrm{hr}$ of goodquality data (following a cut on the satisfactory hardware state of the cameras and good atmospheric conditions) were recorded during 261 runs. The mean zenith angle of these observations were $24^{\circ}$. The new data set B was taken from 2009 to 2010 and consists of 241 runs corresponding to $102 \mathrm{hr}$ of additional exposure. The mean zenith angle of these observations were $23^{\circ}$. The total exposure time (data set A and B) adds up to $213 \mathrm{hr}$. Data set A was taken prior to the launch of the Fermi Gamma-ray Space Telescope, while the new data set B was taken after its launch. The consistency between the results of the H.E.S.S. observations of Cen A in these two time intervals, that is, the lack of flux variability along with no change in spectral parameters, is of importance to substantiate a simultaneous spectral fit of both the HE and VHE data. The Image Pixel-wise fit for Atmospheric Cherenkov Telescope (ImPACT) analysis [9] was used to process the H.E.S.S. data. The std_ImPACT cut configuration, which requires a minimum of 60 photo-electrons per image, was used. The On-source counts were taken from the circular region centred at the Cen A radio core and covering potential VHE emitting regions, such as the (sub)parsec- and kiloparsec-scale jets. The same On-region was selected for analyses of the data sets A and B, and for a combined analysis. The reflected-region background method with multiple Off-source regions was used for spectral measurements.

The re-analysis of data set A yielded a gamma-ray excess of 277 counts above the background, corresponding to a firm detection with a statistical significance of $8.4 \sigma$ following the method of $\mathrm{Li}$ \& $\mathrm{Ma}$ [10]. The increase in significance with respect to the published result in Aharonian et al. [5] is related to the application of improved analysis techniques. The likelihood maximisation for a power-law hypothesis, $d N / d E=N_{0} \times\left(E / E_{0}\right)^{-\Gamma}$, yields a photon index of $\Gamma=2.51 \pm 0.19_{\text {stat }} \pm 0.20_{\text {sys }}$ and a normalisation constant of $N_{0}=\left(1.44 \pm 0.22_{\text {stat }}{ }_{-0.29 \text { sys }}^{+0.43}\right) \times 10^{-13} \mathrm{~cm}^{-2} \mathrm{~s}^{-1} \mathrm{TeV}^{-1}$ at $E_{0}=1 \mathrm{TeV}$.

The analysis of data set B yielded a gamma-ray excess of 245 counts above the background. This gamma-ray excess corresponds to a firm detection with a statistical significance of $8.8 \sigma$. Thus, the Cen A gamma-ray core is clearly detected as a source of VHE emission in both of the H.E.S.S. data sets. The spectral analysis of the data taken in 2009-2010 yields a photon index of $\Gamma=2.55 \pm 0.19_{\text {stat }} \pm 0.20_{\text {sys }}$ and a normalisation constant of $N_{0}=\left(1.50 \pm 0.22_{\text {stat }_{-0.30}}^{+0.45 s}\right) \times 10^{-13}$ $\mathrm{cm}^{-2} \mathrm{~s}^{-1} \mathrm{TeV}^{-1}$ at $E_{0}=1 \mathrm{TeV}$.

To search for variability between the data sets $\mathrm{A}$ and $\mathrm{B}$, one needs to compare the intrinsic spectral properties of the source in these two time intervals. A comparison of the spectral analyses of the H.E.S.S. data sets A and B shows that the values of the spectral parameters are compatible with each other.

Applying the ImPACT analysis to the combined data set, an excess of 522 events above the background is detected. This excess leads to a firm detection of the Cen A gamma-ray core with H.E.S.S. at a statistical significance of $12 \sigma$. The spectral analysis applied to the full data set yields a photon index of $\Gamma=2.52 \pm 0.13_{\text {stat }} \pm 0.20_{\text {sys }}$ and a normalisation constant of $N_{0}=(1.49 \pm$ $0.16_{\text {stat }}^{+0.40_{\text {sys }}}+\times 10^{-13} \mathrm{~cm}^{-2} \mathrm{~s}^{-1} \mathrm{TeV}^{-1}$ at $E_{0}=1 \mathrm{TeV}$. The reconstructed spectrum of the Cen $\mathrm{A}$ gamma-ray core is shown in Fig. 1. All of the eight SED data points in the VHE range are above a $2.5 \sigma$ significance level. The derived data points for each energy band in the VHE range, shown in Fig. 1, agree within error bars with those for the first and second data sets. The VHE spectrum of the Cen A core is compatible with a power-law function $\left(\chi^{2}=3.9\right.$ with 6 DOF).

If one takes the values of the spectral parameters from the LAT four-year Point Source Cata- 


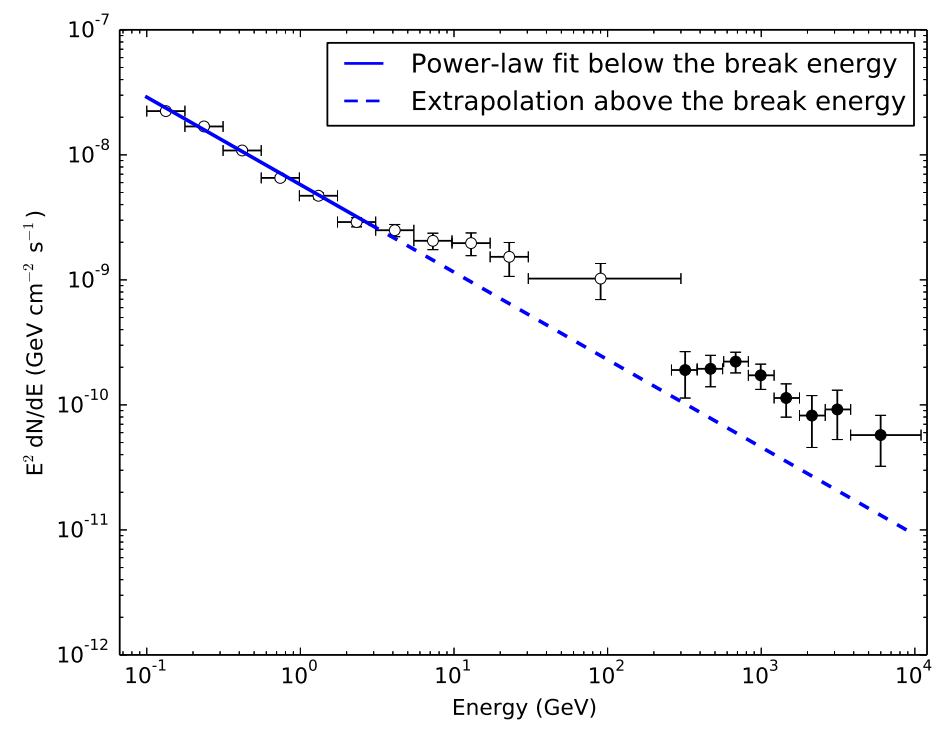

Figure 1: SED of the Cen A $\gamma$-ray core from the paper [3]. Fermi-LAT and H.E.S.S. data points along with a high-energy power-law extrapolation of the $\gamma$-ray spectrum measured below the break energy are shown. Eight years of Fermi-LAT data and 213 hours of H.E.S.S. data were used.

logue (3FGL) [11] obtained from the observations of Cen A between $100 \mathrm{MeV}$ and $100 \mathrm{GeV}$ assuming a single power-law spectrum, then one finds that $N_{0}=(0.45 \pm 0.07) \times 10^{-13} \mathrm{~cm}^{-2} \mathrm{~s}^{-1} \mathrm{TeV}^{-1}$ at $E_{0}=1 \mathrm{TeV}$ and $\Gamma=2.70 \pm 0.03$. Therefore, the differential flux at $1 \mathrm{TeV}$ derived from the H.E.S.S. observations in 2004-2010 is about 3.5 times larger than that inferred from a power-law extrapolation of the 3FGL catalogue spectrum. This indicates that a deviation of the spectrum from a single power law ("hardening") should occur at $\mathrm{GeV}$ energies to match the $\mathrm{TeV}$ data.

\subsection{Fermi-LAT observations and results}

To check the presence of a hardening in the Cen A gamma-ray core at GeV energies, a FermiLAT analysis was performed. In HE gamma rays, the core of Cen A is firmly detected with the Fermi-LAT using eight years of Pass 8 data spanning over three orders of magnitude in energy. Pass 8 SOURCE class Fermi-LAT photon data between August 4, 2008 and July 6, 2016 with energies between $100 \mathrm{MeV}$ and $300 \mathrm{GeV}$ were selected for the analysis. A $10^{\circ} \times 10^{\circ}$ square region of interest (ROI) centred at the position of the Cen A core (3FGL J1325.4-4301) with spatial bins $0.1^{\circ}$ in size is selected. Models are compared based on the maximum value of the logarithm of the likelihood function, $\log \mathcal{L}$. The significance of model components or additional parameters is evaluated using the test statistic, whose expression is TS $=2\left(\log \mathcal{L}-\log \mathcal{L}_{0}\right)$, where $\mathcal{L}_{0}$ is the likelihood of the reference model without the additional parameter or component. For other details on observations and analysis, see the paper [3].

A LAT analysis of Cen A involves unique challenges not present in other individual extragalactic source analyses, largely due to the massive angular extent of the Cen A non-thermal outer lobes of $\sim 9^{\circ}$ [12]. To model the sources within the ROI, sources from the 3FGL within the $15^{\circ} \times 15^{\circ}$ region enclosing the ROI (the 3FGL models the Cen A lobes with a template created from $22 \mathrm{GHz}$ 
WMAP data) are included. Some unmodelled emission from the Cen A lobes was detected and additional background point sources were introduced in order to account for excess lobe emission. These additional background sources correspond to regions with data counts in excess of the model found in the residual TS map that in turn is created by moving a putative point source through a grid of locations on the sky and maximising $\log \mathcal{L}$ at each grid point, with the other, stronger, and presumably well-identified sources included in each fit.

An SED over the full range was computed by dividing the data into 14 equally spaced logarithmic energy bins and then merging the four highest energy bins into one for sufficient statistics. In each bin, the Cen A lobe and core spectral parameters were left free to optimise and within each bin these spectra were fit using a single power law. The resulting SED is plotted in Fig. 1. To plot the data point within the wide merged energy bin, we used the prescription from the reference [13]. From the position of the peak in the likelihood profile (see Fig. 2 from the paper [3]), a best-fit break energy of $2.8_{-0.6}^{+1.0} \mathrm{GeV}$ is found. The spectral hardening in the HE gamma-ray emission from the core of Cen A above an energy break of $2.8 \mathrm{GeV}$ is illustrated in Fig. 1. A broken power-law model describes well the shape of the Fermi-LAT gamma-ray spectrum with a break energy of $2.8 \mathrm{GeV}$. To determine the statistical preference of the broken power-law model over the single power law, we subtract the overall $\log \mathcal{L}$ from the same ROI model with a single power law from the $\log \mathcal{L}$ with the break energy profile at $2.8 \mathrm{GeV}$. Because these models are nested, Wilks' theorem yields a preference of the broken power law with $4.0 \sigma$ confidence $\left(\chi^{2}=19.0\right.$ with 2 DOF). In the lower-energy band, a photon index is of $2.70 \pm 0.02_{\text {stat }_{-0.03}^{+0.05}}^{+\mathrm{s}_{\mathrm{s}} \mathrm{A}_{\mathrm{eff}}}$, and in the higher band, $2.31 \pm 0.07_{\text {stat }}^{+0.01}{ }_{-0.04}$ sys, $A_{\text {eff }}$. This provides corroborating evidence for a spectral hardening by $\Delta \Gamma \sim 0.4$ above the break energy.

To search for variability light curves were computed both above and below the break energy $(2.8 \mathrm{GeV})$ using a single power-law spectral model for each. Below the break the data were divided into 64 45-day bins and above the break the data were divided into nine-month bins. No evidence for flux variability is found in both the analyses.

\subsection{Discussion of the Cen A gamma-ray core}

The smooth $\mathrm{TeV}$ spectrum and the spectral hardening by $\Delta \Gamma \sim 0.4$ as observed with H.E.S.S. and Fermi-LAT are strongly suggestive of the contribution of a second emission component in addition to the conventionally employed single-zone SSC component under the assumption of a misaligned jet. A variety of different (not mutually exclusive) scenarios for the physical origin of this second emission component could be envisaged. Proposals in the literature for Cen A encompass (a) magnetospheric (pulsar-like) scenarios, (b) inner (parsec- and sub-parsec-scale) jet models, (c) extended astrophysical scenarios involving for example hadronic pp-interactions of accelerated protons with ambient matter in its kiloparsec-scale region and (d) explanations involving physics beyond that of the Standard Model, see the paper [3] for references.

While a variety of different interpretations are available, the physical origin of the additional gamma-ray emitting component cannot yet be resolved due to instrumental limitations in angular resolution and the apparent absence of significant variability in both the HE and VHE data. These findings illustrate, however, that radio galaxies such as Cen A are offering important physical insight beyond what could usually be achieved in classical blazar sources. With its increased sensi- 
tivity, Cherenkov Telescope Array [14] is expected to probe deeper into this and help to eventually resolve the nature of the gamma-ray emission in Cen A.

\section{PKS 0625-35}

PKS 0625-354 was observed with H.E.S.S. during eight nights in 2012 November and December, resulting in a total exposure of $5.5 \mathrm{hr}$ of good quality data. The results are published by the H.E.S.S. collaboration on the basis of these observations [4]. Below I give a summary of the paper. All data were taken in wobble mode with the offset of $0.5^{\circ}$. For all the data taken, the observations were between zenith angles of $11^{\circ}-19^{\circ}$.

The data were analysed using the Model Analysis chain [15] with the Loose Cuts configuration. The measured excess of 60.7 events corresponds to $6.1 \sigma$ significance (following [10]). The observed VHE gamma-ray emission is centred on RA $=06^{\mathrm{h}} 26^{\mathrm{m}} 58.2^{\mathrm{s}} \pm 2.6_{\text {stat }}^{s}$ and Dec $=-35^{\circ} 29^{\prime} 50^{\prime \prime} \pm$ $33_{\text {stat }}^{\prime \prime}, \mathrm{J} 2000$. Within the statistical uncertainties, the source exhibits a point-like morphology. The data are fitted with a power-law model with a normalisation of $N_{0}=\left(0.58 \pm 0.22_{\text {stat }} \pm 0.20_{\text {sys }}\right) \times 10^{-12}$ $\mathrm{cm}^{-2} \mathrm{~s}^{-1} \mathrm{TeV}^{-1}$ at $E_{0}=1 \mathrm{TeV}$ and $\Gamma=2.84 \pm 0.50_{\text {stat }} \pm 0.20_{\text {sys. }}$. The goodness of the fit is $\chi^{2} / n_{\text {dof }}=$ $24.4 / 21$. No evidence for variability in VHE gamma-ray emission during the observation period is found.

Having a good multiwavelength coverage from radio to very high energy, a broad-band modelling is performed using two types of emission scenarios, i.e. a one zone lepto-hadronic and a multizone leptonic models. On the grounds of energetics, the analysis favours a leptonic multizone model.

Though the large-scale radio structure of the source is typical of an FR I radio galaxy [16], there is now an accumulated body of evidence for the BL Lac nature of the nucleus and the pc-scale jet. Arguments favouring a blazar or moderately misaligned blazar nature of the source include

(i) the VLBI map from the TANAMI collaboration shows a clearly one-sided pc jet with superluminal apparent velocities [17] of $\beta_{\mathrm{a}}=3.0 \pm 0.5 \mathrm{c}$, requiring a maximum jet misalignment $\theta<37_{-5}^{\circ}$

(ii) Having no counterjet detection, the jet-to-counterjet brightness ratio is $>966$, see Ojha et al. [18]. Given the observed radio spectral index of $\alpha=-0.45$ between 2.7 and $5 \mathrm{GHz}$, the deduced value of the jet misalignment is $\theta<27.6^{\circ}$;

(iii) The flux and frequency of the synchrotron peak, most likely between UV and X-rays, also indicates to a BL Lac nature. This synchrotron peak position, $\log \left(v_{\text {peak }} / \mathrm{Hz}\right) \sim 10^{16}$, is actually the most extreme one amongst all know VHE radio galaxies, as presented in Fig. 8 in [19];

(iv) The redshift of the source $(\mathrm{z}=0.055)$ is very high compared to the other VHE radio galaxies. If strongly misaligned, this would imply an extremely powerful jet. Most of radio galaxies in the centres of cool-core galaxy clusters are weak emitters in gamma rays [20].

(v) Wills et al. [21] have shown that the nuclei of BL Lac type objects and FR I radio galaxies can be used to discriminate the nature following the intensity of their [O III] emission line. The measured [OIII] line of PKS 0625-35 falls within the BL Lac category;

(vi) In order to reproduce the observed multi-wavelength SED, the leptonic and lepto-hadronic models naturally need significant Doppler boosting of the emission $\delta \sim 10-20$, see Tables 4 and 6 [4], associated to small angles with the line of sight. 


\section{Fornax A lobes}

Fornax A along with Centaurus A and M 87 (Virgo A) are the three most luminous radio galaxies located within $50 \mathrm{Mpc}$. Centaurus A and Virgo A are sources firmly detected with H.E.S.S.. Fornax A is located at a distance of $19 \mathrm{Mpc}$ similar to that of M87. However, GeV gamma-ray emission from Fornax A is dominated by its giant lobes of $70 \mathrm{kpc}$ in radius each while $\mathrm{GeV}$ gamma-ray emission from M87 is dominated by the core [22]. Fornax A is at about a 5 times larger distance than that of Centaurus A and therefore the Fornax A giant lobes comfortably fit within the H.E.S.S. field of view. Taking these facts into account, H.E.S.S. observations of the Fornax A giant lobes provide us with an excellent tool to investigate the cosmic ray (CR) particle content of radio galaxy lobes.

The total angular extension of the radio galaxy, Fornax A, is about 1 degree. Fornax A contains two radio lobes with the size of 14 armin in radius each. The distance between the radio lobe centres is about 30 arcmin. The geometrical properties of this radio galaxy provide for the first time an opportunity to perform a morphological analysis of giant lobes in the VHE band.

$\mathrm{GeV}$ gamma-ray emission from Fornax A has been detected with Fermi-LAT at a significance level of about $12 \sigma$ [22]. The spatial extension of this gamma-ray source was studied by comparing the Fermi-LAT data with source model predictions. Comparison between the spatial models, (i) the point source and (ii) the radio lobes template in addition to the point source, established that the $\mathrm{GeV}$ gamma-ray emission from Fornax $\mathrm{A}$ is spatial extended with $6.0 \sigma$ confidence [22]. The Fornax A core contribution was constrained to be less than $14 \%$ of the total gamma-ray flux.

The origin of the high energy gamma rays from the Fornax A giant lobes is a mystery since its discovery by Fermi-LAT. A leptonic model involving scattering of CMB and extragalactic background light photons by energetic electrons within the radio lobes underpredicts the gamma-ray flux by factors of about 2-3. Thus, the conclusion has been drawn that a new gamma-ray-emitting component is required to produce the emission observed with Fermi-LAT and might be due to hadronic emission [22].

A hadronic model involving proton-proton $(p p)$ collisions of CRs with a thermal plasma within the radio lobes is a viable candidate model, but requires a large total CR energy of $10^{61} \mathrm{erg}$ [27]. This CR energy value is very high compared to an estimate of $5 \times 10^{58} \mathrm{erg}$ in the outburst that is assumed to have created the lobes of Fornax A. The excess energy problem may be overcome, however, if the $p p$ collisions are taking place primarily in the filamentary structures of the lobes identified on the VLA 1.4 GHz radio map by Fomalont et al. [23]. This idea supported by McKinley et al. [27] has initially been proposed as an explanation for the gamma-ray emission observed in the so-called Fermi bubbles of our Galaxy (see [24] and [25]). Adiabatic compression of the target gas and magnetic fields into filaments, as suggested by Crocker et al. [25], could produce the observed gamma rays with a total proton energy that is similar to, or less than, the observed thermal energy reported by Seta et al.[26], if the filling factor is small. The CR proton spectrum in these lobes is likely to be power-law up to energies much higher than $100 \mathrm{TeV}$ with the index identical to the photon index derived from the Fermi-LAT data, i.e. $-1.99 \pm 0.07$. Therefore, the hard gamma-ray spectrum is expected to extend into the VHE regime in this scenario. Observations of the Fornax A lobes with H.E.S.S. will allow us to answer the key question if gamma-ray emission from the giant lobes is of hadronic origin. 
Though the origin of ultra-high energy cosmic rays (UHECRs) remains unclear, radio galaxies are amongst the most favourable sources since they satisfy the Hillas criterion. Recently, the results from the Pierre Auger Observatory (PAO) confirmed evidence for anisotropy in the arrival directions of UHECRs on intermediate angular scales [29]. These results lead to a $4 \sigma$ association of UHECR's arrival directions with starburst galaxies and a slightly weaker association with gammaray emitting active galactic nuclei (AGN) from the $2 \mathrm{FHL}$ catalogue. The radio galaxy Fornax A does not appear in the 2FHL catalogue. Matthews et al. [30] showed that including Fornax A in the analysis could explain the observed excess at southern Galactic latitudes in the PAO data, which could increase the significance of the gamma-ray-emitting-AGN association. They suggested that it is more likely to attribute UHECR anisotropies to AGN than to starburst galaxies and that giantlobed radio galaxies such as Centaurus A and Fornax A can explain the PAO data.

In giant-lobed radio galaxies, UHECRs can be accelerated in the nucleus and the kpc-scale jet as well as in the giant lobes, e.g. [28]. Nearby sources of UHECRs up to a ZeV $\left(10^{21} \mathrm{eV}\right)$ could be observed with a multi-messenger approach including secondary gamma rays. Photo-pion and pair production by UHECR protons on the cosmic microwave background results in secondary gammaray fluxes $[31,32]$. The expected spectrum of these gamma rays is hard with photon index of -1.5 making their detection with H.E.S.S. observations more likely than with Fermi-LAT observations.

This, and the possibilities of secondary emission induced by UHECRs can in principle be investigated by future H.E.S.S. observations with an exposure of several tens hours.

\section{Conclusions}

Radio galaxies are an interesting gamma-ray emitting source class allowing us to probe the physics of otherwise "hidden" environments (e.g. close to the black hole or in the jet or lobes).

The recent paper on the Cen A gamma-ray core by the H.E.S.S. and Fermi-LAT collaborations reports results of new (more than $100 \mathrm{hr}$ ) VHE observations with H.E.S.S. accumulated during the Fermi-LAT operation and provides a detailed characterisation of the complete VHE data set using advanced analysis methods. Both the evidence for a spectral hardening in the HE gamma-ray regime and extension into the VHE regime is most naturally interpreted as an indication for the new physical component beyond the conventional SSC component with possible interpretations ranging from black hole magnetospheric processes to astrophysical scenarios involving VHE emission from kiloparsec-scale regions. Both the hardening of the spectrum above the break energy at a few $\mathrm{GeV}$ and the VHE emission excess over the power-law extrapolation of the gamma-ray spectrum measured below the break energy are a unique case amongst the VHE AGN.

The other recent H.E.S.S. collaboration paper on radio galaxies reported the VHE discovery of PKS 0625-35. The arguments favouring a blazar or moderately misaligned blazar nature of the source including significant Doppler boosting of the emission needed to reproduce the VHE spectrum are provided.

Given both the detection of extended gamma-ray emission from the Fornax A lobes with Fermi-LAT in the HE regime and its still unknown nature, future H.E.S.S. observations of this source can provide important information to reveal the physical processes responsible for gammaray emission by studying its extension in the VHE band. 


\section{Acknowledgements}

This work is based on the research supported by the South African Research Chairs Initiative of the Department of Science and Technology and National Research Foundation of South Africa (Grant No 77948). D.A.P. acknowledges support from the Department of Science and Technology/National Research Foundation (DST/NRF) Square Kilometre Array (SKA) post-graduate bursary initiative under the same Grant.

\section{References}

[1] Zech A., Mazin D., Biteau J., Daniel M., Hassan T., Lindfors E., Meyer M. 2019, in Science with the Cherenkov Telescope Array, World Scientific Publishing Co.

[2] Rieger F., Levinson A. 2018, Galaxies, 6, 116

[3] H.E.S.S. Collaboration, Abdalla H., Abramowski A., Aharonian F., Ait Benkhali F., Angüner E. O., Arakawa M., Armand C. et al. 2018, A\&A, 619, id.A71

[4] H.E.S.S. Collaboration, Abdalla H., Abramowski A., Aharonian F., Ait Benkhali F., Akhperjanian A. G., Andersson T., Angüner, E. O. et al., 2018, MNRAS, 476, 4187

[5] H.E.S.S. Collaboration, Aharonian F., Akhperjanian A. G., Anton G., de Almeida U. B., Bazer-Bachi A. R., Becherini Y., Behera B. et al. 2010, ApJ, 695, L40

[6] Urry, C. M., Padovani, P. 1995, PASP, 107, 803

[7] Reimer A., Böttcher M. 2013, Astropart. Phys., 43, 103

[8] Sahakyan N., Yang R., Aharonian F. A., Rieger, F. M. 2013, ApJL, 770, L6

[9] Parsons R. D., Hinton J. A. 2014, Astropart. Phys., 56, 26

[10] Li T.-P., Ma Y.-Q. 1983, ApJ, 272, 317

[11] Fermi-LAT Collaboration, Acero F., Ackermann M., Ajello M., Albert A., Atwood W. B., Axelsson M., Baldini L. et al., 2015, ApJS, 218, 23A

[12] Fermi-LAT Collaboration, Abdo A. A., Ackermann M., Ajello M., Atwood W. B., Baldini L., Ballet J., Barbiellini G. 2010, Science, 328, 725

[13] Lafferty G. D., Wyatt T. R., 1995, NIMPA, 355, 541

[14] CTA Consortium 2019, Science with the Cherenkov Telescope Array, World Scientific Publishing Co.

[15] de Naurois M., Rolland L. 2009, Astropart. Phys., 32, 231

[16] Ekers R. D., Wall J. V., Shaver P. A., Goss W. M., Fosbury R. A. E., Danziger I. J., Moorwood A. F. M. et al. 1989, MNRAS, 236, 737

[17] Müller C., Krauss F., Kadler M., Trüstedt J., Ojha R., Ros E., Wilms J. et al. 2013, preprint:1310.4384

[18] Ojha R., Kadler M., Böck M., Booth R., Dutka M. S., Edwards P. G., Fey, A. L. et al. 2010, A\&A, 519, A45

[19] Fukazawa Y., Finke J., Stawarz ÅA., Tanaka Y., Itoh R., Tokuda S. 2015, ApJ, 798, 74

[20] Prokhorov D. A., Churazov E. M. 2014, A\&A, 576, A93

[21] Wills K. A., Morganti R., Tadhunter C. N., Robinson T. G., Villar-Martin M. 2004, MNRAS, 347, 771 
[22] Fermi-LAT Collaboration, Ackermann M., Ajello M., Baldini L., Ballet J., Barbiellini G., Bastieri D., Bellazzini R. et al., 2016, ApJ, 826, 1

[23] Fomalont E. B., Ebneter K. A., van Breugel W. J. M., Ekers R. D., 1989, ApJ, 346, 17

[24] Crocker R. M., Aharonian F., 2011, Phys. Rev. Lett., 106, 101102

[25] Crocker R. M., Bicknell G. V., Carretti E., Hill A. S., Sutherland R. S. 2014, ApJL, 791, L20

[26] Seta H., Tashiro M. S., Inoue S. 2013, PASJ, 65, 106

[27] McKinley B., Yang R., López-Caniego, M., Briggs F., Hurley-Walker N., Wayth R. B., Offringa, A. R. et al. 2015, MNRAS, 446, 3478

[28] Hardcastle M. J. 2010, MNRAS, 405, 2810

[29] The Pierre Auger collaboration 2018, ApJL, 853, L29

[30] Matthews J. H., Bell A. R., Blundell K. M., Araudo A. T. 2018, MNRAS, 479L, 76

[31] Armengaud E., Sigl G., Miniati F. 2006, Phys. Rev. D, 73, 083008

[32] Decerprit G., Allard D. 2011, A\&A, 535, A66 